\title{
Movilización y utilización de los sustratos energéticos de Arca zebra (Bivalvia: Arcidae) en relación con el ambiente
}

\section{Mobilization and utilization of energy substrates of Arca zebra (Bivalvia: Arcidae) in regards to the environment}

\author{
José A. Villarroel-Moreno ${ }^{1}$, Vanessa Acosta-Balbas ${ }^{1 *}$ y Miguel A. Guevara-Acosta ${ }^{2}$
}

\begin{abstract}
RESUMEN
El análisis de los componentes bioquímicos en especies marinas para el consumo humano tiene una gran importancia, ya que permite conocer las propiedades alimenticias y energéticas del organismo. En el presente estudio, se evaluó el perfil bioquímico en tejidos de Arca zebra en relación con los factores ambientales, con la finalidad de ser utilizado como indicador de la dinámica reproductiva y de los periodos de engorde de este importante recurso pesquero. Se analizaron 20 organismos/ mes, entre julio de 2010-julio de 2011. A cada organismo se le determinó el largo total (LT) y se separaron los tejidos, los cuales se llevaron hasta peso constante para realizarles los análisis bioquímicos mediante espectrofotometría. Se establecieron diferencias mensuales en la LT, con un promedio anual de $69.61 \mathrm{~mm}$, manteniéndose por encima de la talla comercial. Los carbohidratos y las proteínas mostraron diferencias entre meses, con valores máximos en marzo y julio de 2011, siendo el músculo quien registró el mayor contenido de ambos sustratos energéticos, asociados a los altos niveles de clorofila $a$. Se observó una relación inversa entre los carbohidratos y las proteínas en los tres tejidos con respecto a la masa gonadal, sugiriendo una posible transferencia durante el proceso reproductivo de A. zebra. En las gónadas se registró el mayor contenido de lípidos, sin variación mensual, debido a la sincronía reproductiva de la especie. A. zebra muestra su mejor condición nutricional durante los periodos comprendidos entre septiembre-diciembre y marzo-junio, en estos meses se recomienda su comercialización e ingesta.
\end{abstract}

Palabras claves: Arcidae, componentes bioquímicos, factores ambientales (temperatura, seston, $\mathrm{Cl} a$ ), Venezuela, reproducción.

\begin{abstract}
The analysis of biochemical components in marine species for human consumption is of great importance because it allows knowing the nutritional and energy properties of the organism. The biochemical profile of Arca zebra tissues was evaluated in this research in relation to environmental factors, with the purpose of using this information as an indicator of the reproductive dynamics and fattening periods of this important fishery resource. A total of 20 organisms were analyzed per month between July 2010 and July 2011. Total length (TL) was determined in each organism, and tissues were separated using spectrophotometry, leaving them at a constant weight

\footnotetext{
Departamento de Biología, Núcleo de Sucre, Universidad de Oriente. Cumaná, Estado Sucre, C.P. 6101, Venezuela. vanessaacosta@yahoo.com*

2 Laboratorio de Biotecnología de Microalgas, Departamento Biología Pesquera, Instituto Oceanográfico de Venezuela. Universidad de Oriente, Venezuela.
}

Recibido: 4 de junio de 2015

Corregido: 15 de octubre de 2015

Aceptado: 24 de octubre de 2015

DOI: http://dx.doi.org/10.15359/revmar.8-1.5

Rev. Mar. Cost. ISSN 1659-455X. Vol. 8 (1): 63-78, Enero-Junio 2016. 
for biochemical analyzes. TL differences were established monthly, with an annual average of $69.61 \mathrm{~mm}$, staying above commercial size. Differences were detected for carbohydrates and proteins between months, with maximum values in March and July 2011, being the muscle the part with the highest content of both energy substrates, related to high levels of chlorophyll $a$. An inverse relationship was observed between carbohydrates and proteins in the three tissues in regards to the gonadal mass, suggesting a possible transfer during the reproductive process of $A$. zebra. The greatest lipid content without monthly variation was recorded in gonads due to the species reproductive synchrony. A. zebra shows its best nutritional condition during the periods from September to December and March to June. Commercialization and consumption is recommended during those months.

Keywords: Arcidae, biochemical components, environmental factors (temperature, seston, $\mathrm{Cl} a$ ), Venezuela, reproduction.

\section{INTRODUCCIÓN}

Los tejidos somáticos $\mathrm{y}$ germinales de los moluscos bivalvos experimentan variaciones temporales que reflejan la disponibilidad de energía en el transcurso del tiempo según sus necesidades fisiológicas y reproductivas. En tal sentido, el metabolismo en moluscos bivalvos (mantenimiento basal, crecimiento y reproducción) está relacionado con la movilización y consumo de energía, ya sea obtenida directamente del alimento ingerido o de las reservas almacenadas en los órganos especializados, tales como: glándula digestiva, músculo aductor y manto (Gabbott, 1983; Pérez et al. 2003). Según Acosta et al. (2010), la composición química de los tejidos de dichos organismos determina el contenido energético de su carne y este a su vez puede seguir diferentes vías, como: 1) utilización inmediata, 2) almacenamiento en órganos especializados y/o 3) utilización y almacenamiento, lo que dependerá de los requerimientos fisiológicos y metabólicos del organismo en un momento determinado.
Arca zebra (Swainson, 1883) es un bivalvo que pertenece a la familia Arcidae, se distribuye desde las costas del Golfo de México y sur de Florida hasta el norte de Brasil. En Venezuela se localiza en la costa este de la isla de Margarita, en las costas occidentales de la península de Araya, en la costa norte del golfo de Cariaco y en la costa occidental del Estado Falcón (Arias, 1976). Particularmente, en el nororiente de Venezuela forma un extenso banco natural, siendo el principal centro de extracción pesquera el ubicado entre CocheChacopata, considerado como uno de los más productivos del mundo y al cual están asociados una gran variedad de moluscos bivalvos de importancia comercial (Mendoza, 1999; Acosta et al. 2007; Mendoza, 2008).

Debido al gran interés comercial de $A$. zebra, se hace necesario conocer cómo es la movilización de los sustratos energéticos en relación con el ambiente donde se encuentra, con el fin de poder establecer lineamientos para su explotación racional que garanticen el uso sustentable de este bivalvo en el nororiente de Venezuela. 
Hasta el momento solo se han llevado a cabo estudios sobre la composición proximal de $A$. zebra en el organismo completo y como fuente de información nutricional o en relación con la fijación, crecimiento y algunos aspectos tecnológicos en esta especie (Arias, 1976; Acuña, 1977; Cabello et al. 2004). Los trabajos sobre la movilización de sustratos energéticos en los tejidos de $A$. zebra son escasos y la única información existente al respecto es el trabajo realizado en las Bermudas por Sarkis (1993), quien evaluó los cambios estacionales en la composición bioquímica de esta especie. Por tal motivo, en el presente estudio, se evaluó el perfil bioquímico en tres tejidos (somáticos y reproductivos) de A. zebra y su relación con los cambios ambientales, procesos reproductivos y engorde, con la finalidad de conocer las estrategias o mecanismos de transferencia de energía que emplea esta especie, que ayuden a establecer políticas de aprovechamiento y conservación de este importante recurso pesquero.

\section{MATERIALES Y MÉTODOS Obtención de las muestras}

Los muestreos se realizaron mensualmente entre julio de $2010 \mathrm{y}$ julio de 2011 en el banco natural de $A$. zebra, ubicado en Chacopata entre los estados Sucre y Nueva Esparta, en el nororiente de Venezuela $\left(10^{\circ} 42^{\prime}-10^{\circ}\right.$ $\left.46^{\prime} \mathrm{Ny} 63^{\circ} 46^{\prime}-63^{\circ} 54^{\prime} \mathrm{O}\right)$. Las capturas se llevaron a cabo empleando una rastra de $120 \times 86 \mathrm{~cm}$ a una profundidad aproximada de $8 \mathrm{~m}$, manipulada desde una embarcación y siguiendo los lineamientos establecidos en la resolución MAC-RNR-266 de fecha 02/09/1960 para la extracción de $A$. zebra (llamada localmente "pepitona"). Los ejemplares recolectados fueron colocados en contenedores isotérmicos y de inmediato llevados al laboratorio para su refrigeración $\left(-7^{\circ} \mathrm{C}\right)$ y para los análisis posteriores.

\section{Factores ambientales}

Las muestras de agua de mar en la zona de captura fueron recolectadas quincenalmente con un cubo plástico de $6 \mathrm{~L}$ de capacidad. A partir de estas muestras se tomaron $2 \mathrm{~L}$ de agua para determinar la concentración de clorofila $a$ y seston total (Strickland \& Parsons, 1972). La temperatura se registró in situ utilizando un termómetro $\left( \pm 0.1^{\circ} \mathrm{C}\right)$.

\section{Procesamiento de las muestras}

En el laboratorio, a cada ejemplar (20 organismos/mes) se le eliminaron todos los epibiontes. Luego se le midió la longitud de la concha (LT) con un vernier digital (Mitutoyo $\pm 0.01 \mathrm{~mm}$ ). Seguidamente, los organismos fueron disectados para separar el músculo, las gónadas y el resto de tejidos, los cuales fueron colocados en una estufa (marca Memmert) a $60{ }^{\circ} \mathrm{C} / 48 \mathrm{~h}$ hasta obtener masa constante, empleando una balanza analítica marca Denver, modelo TR204 de $0.001 \mathrm{~g}$ de apreciación.

\section{Análisis bioquímicos}

Las determinaciones de los contenidos totales de carbohidratos, proteínas y lípidos se llevaron a cabo, por duplicado, en grupos de 10 individuos tomados al azar. La cuantificación de 
los carbohidratos se hizo por el método fenol sulfúrico (Dubois et al. 1956), se contrastó con una curva patrón de solución de glucosa (D-Glocusa; SIGMA, G-5767). La absorbancia fue medida a $490 \mathrm{~nm}$. Las proteínas fueron analizadas empleando el colorante hidrofóbico Coomassie Brilliant Blue G-250, CBB (Bradford, 1976), usando una curva patrón de Sero albúmina $(0.02 \mathrm{mg} / \mathrm{mL})(S I G M A, T 5888)$, la absorbancia se midió a $595 \mathrm{~nm}$. Los lípidos fueron extraídos de acuerdo con el protocolo de Bligh \& Dyer (1959), cuantificados según Pande et al. (1963), utilizando una curva patrón con una disolución de tripalmitina (2 $\mathrm{mg} / \mathrm{mL}$ ) y midiendo la absorbancia a $590 \mathrm{~nm}$. Los resultados de las biomoléculas fueron expresados en $\mathrm{mg} / \mathrm{g}$ de materia orgánica libre de cenizas.

\section{Análisis estadísticos}

Las diferencias mensuales en la biomasa y los componentes bioquímicos de cada tejido de $A$. zebra fueron analizadas mediante la aplicación de un ANOVA de un factor, previo cumplimiento de los supuestos de normalidad y varianza. En los casos donde se detectaron diferencias significativas $(P<0.05)$ se aplicó una prueba a posteriori de Scheffè (Zar, 2010). Para estimar la posible influencia de la temperatura, la biomasa fitoplanctónica y el seston total sobre la composición bioquímica se realizó un análisis deregresiónmúltiple "Stepwise", según Hair et al. (1992). Finalmente, para evaluar la posible relación existente entre la masa de la gónada como indicadora de actividad reproductiva y los componentes bioquímicos se realizó un análisis de correlación de Spearman. Los análisis estadísticos se efectuaron con el programa Statgraphic Centurium XV (Statpoint, 2006).

\section{RESULTADOS}

Talla: El patrón biométrico de $A$. zebra representado por la LT mostró diferencias significativas $(\mathrm{Fs}=$ 33.96; $P<0.05$ ). Las tallas menores se obtuvieron en los meses de julio de $2010(\mathrm{LT}=59.93 \pm 9.47 \mathrm{~mm})$ y marzo de $2011(\mathrm{LT}=60.65 \pm 8.05$ $\mathrm{mm}$ ), mientras que en septiembre se registraron valores mayores (LT= $79.78 \pm 4.58 \mathrm{~mm}$ ). Se observaron otros aumentos importantes en los meses de diciembre de 2010, febrero, mayo y julio de 2011 (Fig. 1).

Masa seca: La masa del resto de tejidos (Fig. 2A) presentó diferencias significativas $(\mathrm{Fs}=21.68 ; P<0.05)$ entre los meses de muestreo. En septiembre y diciembre de 2010 (2.03 $\pm 0.46 \mathrm{~g}$ y $1.54 \pm 0.33 \mathrm{~g}$ ), febrero y mayo de $2011(1.60 \pm 0.44 \mathrm{~g}$ y 1.67 $\pm 0.50 \mathrm{~g}$ ), se obtuvieron las masas más altas, mientras que las mínimas se registraron en julio de $2010(0.95$ $\pm 0.39 \mathrm{~g})$ y marzo de $2011(0.94 \pm$ $0.27 \mathrm{~g}$ ). La masa seca muscular (Fig. 2B) también presentó diferencias significativas entre los meses de muestreo $(\mathrm{Fs}=15.17 ; P<0.05)$, alcanzando los valores mínimos en julio de $2010(0.20 \pm 0.11 \mathrm{~g})$ y marzo de $2011(0.20 \pm 0.08 \mathrm{~g})$ y los valores máximos en septiembre y diciembre de $2010(0.51 \pm 0.13$ y $0.39 \pm 0.11 \mathrm{~g})$, así como en febrero y mayo de 2011 $(0.39 \pm 0.15$ y $0.39 \pm 0.17 \mathrm{~g})$. El tejido 


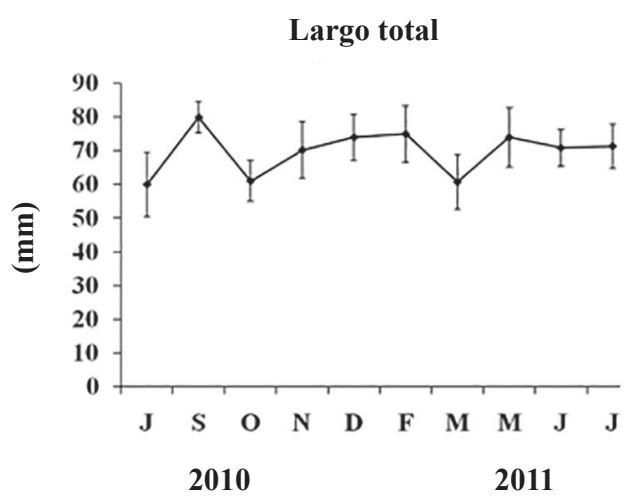

Fig. 1. Variación mensual del largo total (LT) de Arca zebra proveniente de Chacopata, durante el periodo julio 20102011. Las líneas verticales muestran la desviación estándar con respecto a la media Fig. 1. Monthly variation of total length (LT) of Arca zebra from Chacopata during July 2010-2011. Vertical lines show the standard deviation from the mean

gonádico (Fig. 2C) mostró variaciones durante todo el periodo de muestreo $(\mathrm{Fs}=10.80 ; P<0.05)$, apreciándose en septiembre aumentos $(0.79 \pm 0.48 \mathrm{~g})$ y luego presentó un nuevo incremento en diciembre de $2010(0.56 \pm 0.33$ g) y mayo de $2011(0.49 \pm 0.28 \mathrm{~g})$, con disminución de los valores entre dichos meses, las fluctuaciones se mantuvieron hasta el final del estudio.

Factores ambientales: $\mathrm{La}$ temperatura osciló de 27 a $30{ }^{\circ} \mathrm{C}$ entre julio y noviembre de 2010 (Fig. 3A), luego descendió progresivamente hasta alcanzar valores mínimos en febrero y marzo de 2011 (23.80 \pm 0.28 y $23.85 \pm 0.07{ }^{\circ} \mathrm{C}$ ), para luego presentar incrementos desde $24^{\circ} \mathrm{C}$ hasta $28^{\circ} \mathrm{C}$ en mayo, manteniéndose hasta julio de 2011 donde se observó un pequeño descenso. La biomasa
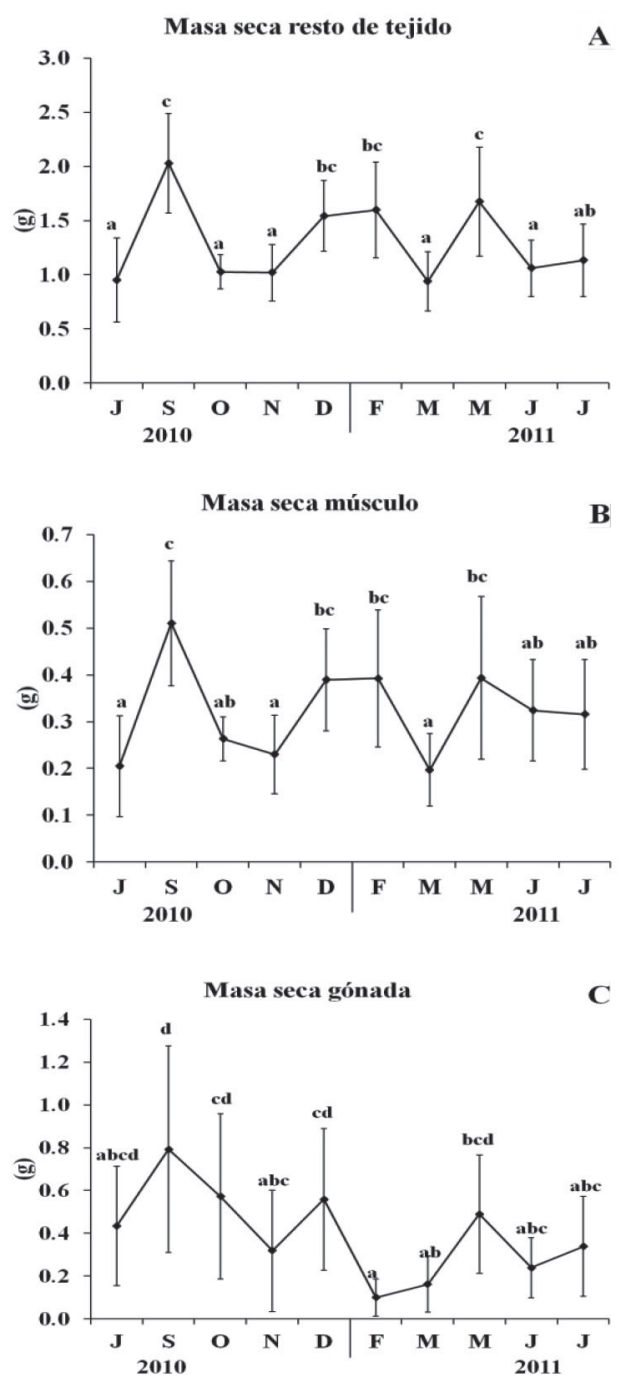

Fig. 2. Variación mensual de la masa seca del resto de tejido (A), músculo (B) y gónada (C) de Arca zebra proveniente de Chacopata, durante el periodo julio 2010-2011. Las líneas verticales muestran la desviación estándar con respecto a la media. Meses con letras iguales no presentan diferencias significativas $(P>0.05)$

Fig. 2. Monthly variation of the dry mass of the remaining tissue (A), muscle (B) and gonads (C) of Arca zebra from Chacopata during July 2010-2011. Vertical lines show the standard deviation from the mean. Months with the same letter present no significant differences $(P>0.05)$ 
fitoplanctónica estimada por clorofila $a$ (Fig. 3B) mostró valores por encima de $1 \mu \mathrm{g} / \mathrm{L}$, con un promedio inicial en julio de $2010(3.06 \pm 1.04 \mu \mathrm{g} / \mathrm{L})$, descendió en septiembre $(1.31 \pm 0.97$ $\mu \mathrm{g} / \mathrm{L}$. Posteriormente, se produjo un aumento paulatino hasta marzo de $2011(3.63 \pm 2.32 \mu \mathrm{g} / \mathrm{L})$ y un descenso brusco en mayo $(1.17 \pm 0.55 \mu \mathrm{g} / \mathrm{L})$. Un segundo aumento se evidencia en junio de $2011(3.90 \pm 3.82 \mu \mathrm{g} / \mathrm{L})$. El seston total (Fig. 3C) no mostró un patrón definido, mostrando al inicio del estudio una concentración de $15.91 \pm 1.29 \mathrm{mg} / \mathrm{L}$ (julio de 2010) para luego descender bruscamente hasta diciembre de $2010(1.59 \pm 0.11$ $\mathrm{mg} / \mathrm{L}$ ), a partir de este mes comienza a incrementar hasta alcanzar un valor máximo en mayo de 2011 (21.71 \pm $5.37 \mathrm{mg} / \mathrm{L}$ ), donde se evidencia una segunda disminución hasta julio de $2011(7.67 \pm 1.07 \mathrm{mg} / \mathrm{L})$.

\section{Análisis bioquímicos}

Proteínas: El contenido de proteínas en el resto de tejido, músculo y gónadas (Fig. 4) mostró diferencias significativas entre los meses de muestreo $(\mathrm{Fs}=15.61 ; \mathrm{Fs}=$ 10.21; Fs $=9.21 ; P<0.05)$. Todos los tejidos analizados presentaron sus máximos contenidos de proteínas en junio de 2011 (437.93 \pm 21.87 ; $485.37 \pm 37.05$ y $356.54 \pm 24.60 \mathrm{mg} / \mathrm{g}$, respectivamente). En el caso del resto de tejido (Fig. 4A), los valores más bajos se presentaron en septiembre-octubre de $2010(287.52 \pm 45.70$ y $286.82 \pm$ $20.84 \mathrm{mg} / \mathrm{g}$, respectivamente). Para el músculo (Fig. 4B) se obtuvieron en el lapso septiembre-diciembre de

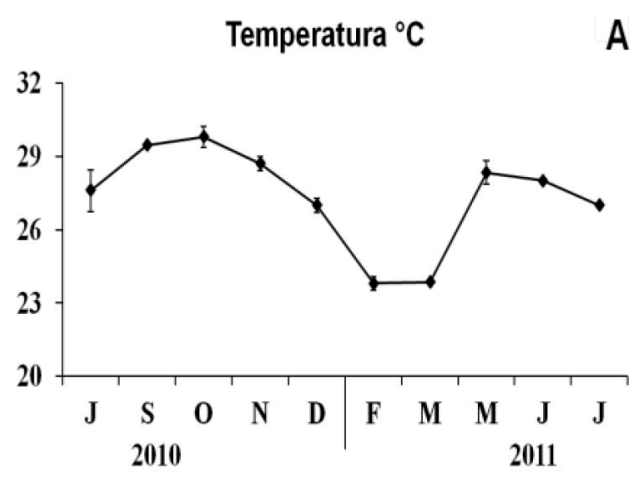

Clorofila $a(\mu \mathrm{g} / \mathrm{L})$

B

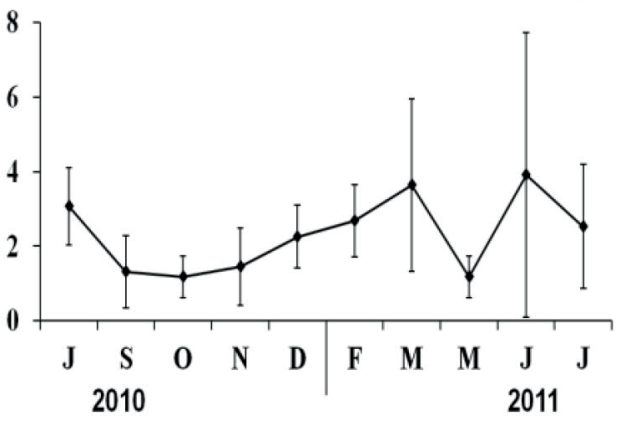

Seston total $(\mathrm{mg} / \mathrm{L})$

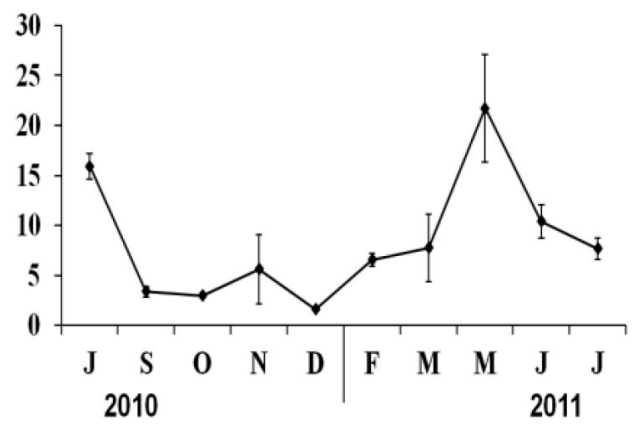

Fig. 3. A) Variación mensual de la temperatura $\left({ }^{\circ} \mathrm{C}\right)$, B) Clorofila $a(\mu \mathrm{g} / \mathrm{L})$ y C) Seston total (mg/L) del banco natural de Arca zebra (Chacopata), durante el periodo julio 20102011. Las líneas verticales muestran la desviación estándar con respecto a la media Fig. 3. A) Monthly variation of temperature $\left({ }^{\circ} \mathrm{C}\right)$, B) Chlorophyll $a(\mu \mathrm{g} / \mathrm{L})$ and C) Total Seston $(\mathrm{mg} / \mathrm{L})$ of the natural bank of Arca zebra (Chacopata) during July 2010-2011. Vertical lines show the standard deviation from the mean 

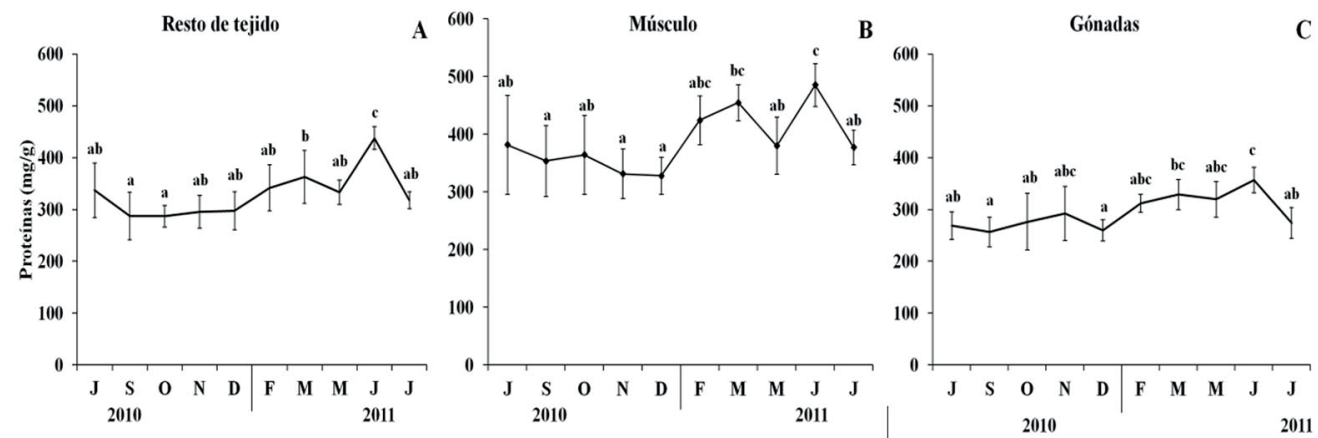

Fig. 4. Variación mensual de proteínas totales en A) resto de tejido, B) músculo y C) gónadas de Arca zebra. Las líneas verticales muestran la desviación estándar con respecto a la media. Meses con letras iguales no presentan diferencias significativas $(P>0.05)$

Fig. 4. Monthly variation of total proteins in A) remaining tissue, B) muscle and C) gonads of Arca $z e b r a$. Vertical lines show the standard deviation from the mean. Months with the same letter present no significant differences $(P>0.05)$
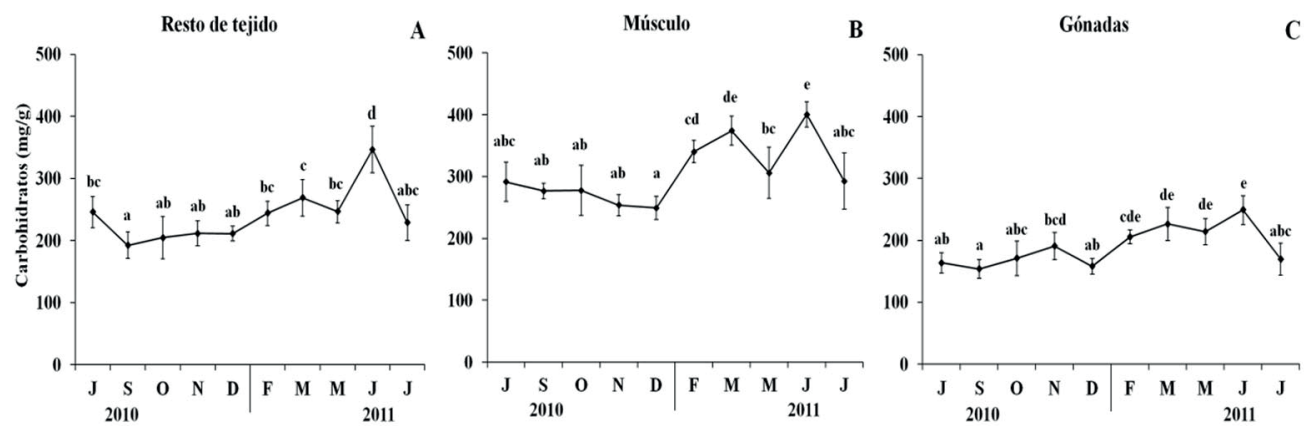

Fig. 5. Variación mensual de carbohidratos totales en A) resto de tejido, B) músculo y C) gónadas de Arca zebra. Las líneas verticales muestran la desviación estándar con respecto a la media. Meses con letras iguales no presentan diferencias significativas $(P>0.05)$

Fig. 5. Monthly variation of total carbohydrates in A) remaining tissue, B) muscle and C) gonads of Arca zebra. Vertical lines show the standard deviation from the mean. Months with the same letter present no significant differences $(P>0.05)$

2010: $353.55 \pm 61.16 ; 363.83 \pm 68.61$ $331.21 \pm 42.89$ y $327.84 \pm 32.28 \mathrm{mg} / \mathrm{g}$, respectivamente. En el tejido gonádico (Fig. 4C), los mínimos valores se produjeron en septiembre y diciembre de $2010(256.63 \pm 28.36$ y $259.79 \pm$ $20.41 \mathrm{mg} / \mathrm{g}$, respectivamente).

Carbohidratos: Loscarbohidratos totales en el resto de tejido, músculo y gónadas (Fig. 5) mostraron variaciones mensuales $(\mathrm{Fs}=24.20 ; \mathrm{Fs}=29.61 ; \mathrm{Fs}=$ 22.53; $P<0.05$, respectivamente). Estas macromoléculas presentaron un comportamiento similar al descrito para las proteínas, en todos los tejidos analizados el valor más alto se tuvo en junio de 2011 (346.79 \pm 37.72 ; $400.70 \pm 20.54$ y $249.11 \pm 23.34$ $\mathrm{mg} / \mathrm{g}$, respectivamente), con valores mínimos en septiembre de 2010 para el 

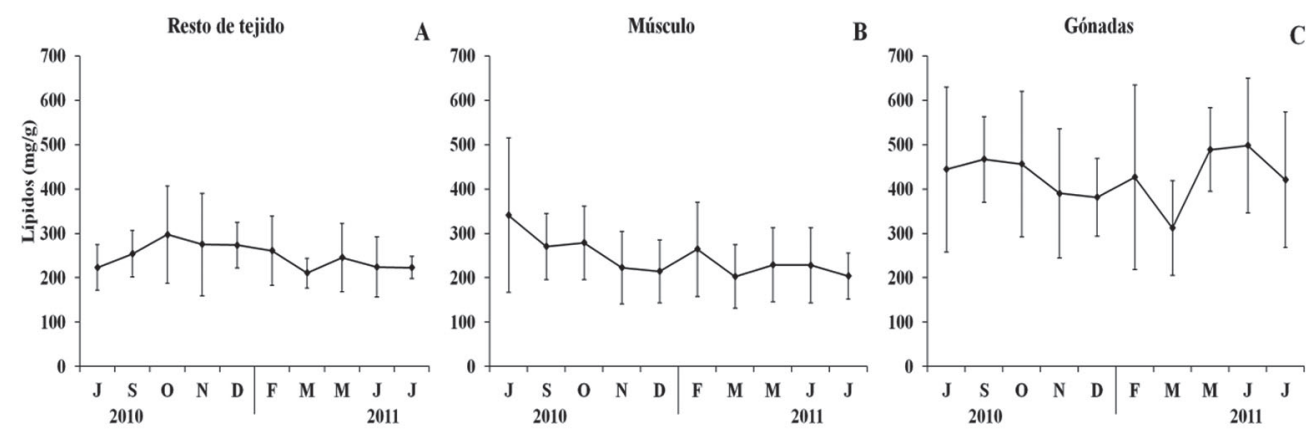

Fig. 6. Variación mensual de lípidos totales en A) resto de tejido, B) músculo y C) gónadas de Arca zebra. Las líneas verticales muestran la desviación estándar con respecto a la media

Fig. 6. Monthly variation of total lipids in A) remaining tissue, B) muscle and C) gonads of Arca zebra. Vertical lines show the standard deviation from the mean

resto de tejido (Fig. 5A) y las gónadas (Fig. 5C) (192.52 $\pm 21.51 ; 153.75 \pm$ $15.24 \mathrm{mg} / \mathrm{g}$, respectivamente) y en diciembre de 2010 para el músculo (Fig. 5B) (249.50 $\pm 18.65 \mathrm{mg} / \mathrm{g})$.

Lípidos: Los niveles de lípidos del resto de tejido, músculo y gónadas (Fig. 6) no presentaron diferencias significativas entre los meses $(\mathrm{Fs}=0.78$; $\mathrm{Fs}=1.07 ; \mathrm{Fs}=0.75 ; P>0.05)$. A pesar de esto, los lípidos del resto de tejido (Fig. 6A) mostraron dos comportamientos: de julio-diciembre de 2010 se observó una tendencia al aumento, mientras que entre febrero-julio de 2011 se produjo una disminución. En el músculo (Fig. 6B) se pudo apreciar una disminución progresiva de lípidos desde julio de 2010 con el máximo valor registrado $(341.04 \pm 174.8$ $\mathrm{mg} / \mathrm{g})$ hasta diciembre de $2010(214.14 \pm$ $71.26 \mathrm{mg} / \mathrm{g}$ ), incrementándose luego en febrero de 2011 (264.02 $\pm 106.19 \mathrm{mg} / \mathrm{g})$ y manteniéndose relativamente estable el resto de los meses. En el caso de las gónadas (Fig. 6C), a partir de julio de 2010 $(444.14 \pm 185.91 \mathrm{mg} / \mathrm{g})$ se observó una disminución, registrando el valor mínimo en marzo de 2011 (312.06 \pm 106.85 $\mathrm{mg} / \mathrm{g}$ ) con tendencia a incrementar, alcanzando un valor máximo en junio de 2011 (497.65 $\pm 151.83 \mathrm{mg} / \mathrm{g})$.

\section{Relación de factores ambientales} con los componentes bioquímicos: $\mathrm{El}$ análisis de regresión múltiple Stepwise, aplicado a cada uno de los componentes bioquímicos de cada tejido (Cuadro 1), mostró que la clorofila $a$ fue el único factor ambiental que aportó porcentajes de explicación de la varianza observada con respecto a los diferentes sustratos energéticos en los tejidos analizados. En el caso de las proteínas, la clorofila $a$ explicó hasta un $65.8 \%$ (resto de tejido) y un $61.5 \%$ (músculo) de la varianza observada, mientras que para los carbohidratos dicho factor ambiental aporta hasta el $61.5 \%$ (resto de tejido) y un $57.4 \%$ (músculo) de la varianza observada. En cuanto a los lípidos, la clorofila $a$ solo pudo explicar de manera inversa y significativa el $53.8 \%$ de la varianza observada en el resto de tejido. 
Cuadro 1. Análisis de regresión múltiple por etapas (Stepwise) aplicado a las proteínas, los carbohidratos y los lípidos del resto de tejidos, gónadas y músculo de Arca zebra con respecto a las variables ambientales (clorofila $a$, seston total y temperatura). DE: desviación estándar, F: frecuencia, $\mathrm{r}^{2}$ : coeficiente de determinación, P: probabilidad Table 1. Stepwise Regression Analysis applied to proteins, carbohydrates and lipids in the remaining tissues, gonads and muscle of Arca zebra with respect to environmental variables (chlorophyll $a$, total seston, and temperature). SD: standard deviation, F: frequency, $\mathrm{r}^{2}$ : determination coefficient, $\mathrm{P}$ : probability

\begin{tabular}{llllll}
\hline Componentes bioquímicos & Coeficiente & $\boldsymbol{D E}$ & $\boldsymbol{F}$ & $\boldsymbol{r}^{2}$ & $\boldsymbol{P}$ \\
\hline $\begin{array}{l}\text { Proteínas } \\
\text { Resto de tejidos (clorofila } a \text { ) }\end{array}$ & 36.53 & 9.31 & 15.37 & 0.65 & $<0.01$ \\
$r^{2}=0.65 ; \mathrm{n}=10 ; F_{1.6}=15.37 ; P<0.01$ & & & & & \\
$\quad \begin{array}{l}\text { Músculo (clorofila } a \text { ) } \\
r^{2}=0.65 ; \mathrm{n}=10 ; F_{1.6}=15.37 ; P<0.01\end{array}$ & 39.73 & 11.11 & 12.78 & 0.61 & $<0.01$ \\
Carbohidratos & & & & & \\
$\begin{array}{l}\text { Resto de tejidos (clorofila } a \text { ) } \\
r^{2}=0.61 ; \mathrm{n}=10 ; F_{1.6}=12.80 ; P<0.01\end{array}$ & 34.01 & 9.50 & 12.80 & 0.61 & $<0.01$ \\
$\begin{array}{l}\text { Músculo (clorofila } a \text { ) } \\
r^{2}=0.57 ; \mathrm{n}=10 ; F_{1.6}=10.76 ; P<0.05\end{array}$ & 37.33 & 11.37 & 10.76 & 0.57 & $<0.05$ \\
$\begin{array}{l}\text { Lípidos } \\
\text { Resto de tejidos }(\text { clorofila } a \text { ) }\end{array}$ & & & & & \\
$r^{2}=0.53 ; \mathrm{n}=10 ; F_{16}=9.30 ; P<0.05$ & -20.35 & 6.67 & 9.30 & 0.53 & $<0.05$ \\
\hline
\end{tabular}

Cabe destacar que ningún sustrato energético analizado en la gónada mostró relación alguna con respecto a los factores ambientales analizados.

Análisis de correlación: En el Cuadro 2 se muestra la correlación de Spearman entre la masa seca gonadal y los componentes bioquímicos de cada tejido analizado, pudiéndose observar la existencia de una relación negativa y significativa entre los niveles de proteínas totales del resto de tejido, gónadas y músculo con la masa seca gonadal $(r=-0.78 ;-0.70$ y $-0.67 ; P<$ 0.05 , respectivamente). Al igual que en el sustrato energético anterior, los carbohidratos totales de los distintos tejidos mostraron también una relación inversa y significativa $(P<0.05)$ con la masa seca gonadal de $r=-0.79$ en el caso del resto de tejido, $r=70.91$ para las gónadas y de $r=-0.67$ en el caso de los carbohidratos del músculo, por último, con respecto a los lípidos, estos no presentaron una relación positiva ni negativa significativa con la masa seca gonadal. La relación más alta observada fue de $r=0.4182$ entre los lípidos del músculo y la masa gonadal.

\section{DISCUSIÓN}

La estructura poblacional de A. zebra se mantuvo relativamente estable durante todo el estudio con un promedio anual de $69.61 \mathrm{~mm}$ de longitud, coincidiendo estos valores con otros estudios realizados en la zona, como los de Prieto \& Saint- 
Cuadro 2. Análisis de correlación (Spearman) entre la biomasa seca gonadal y los componentes bioquímicos (proteínas, carbohidratos y lípidos) en cada uno de los tejidos analizados (resto de tejido, gónada y músculo) de Arca zebra ( $\mathrm{n}=10$ meses)

Table 2. Spearman' Correlation Analysis between gonadal dry biomass and biochemical components (proteins, carbohydrates and lipids) in each of the tissues tested (other tissue, muscle, and gonads) of Arca zebra ( $\mathrm{n}=10$ months)

\begin{tabular}{cccccccccc}
\hline & Gónada & P.R.T. & P.G. & P.M. & C.R.T. & C.G. & C.M. & L.R.T. & L.G. L.M. \\
\hline Gónada & 1 & & & & & & & & \\
P.R.T. & -0.78 & 1 & & & & & & & \\
& $0.02 *$ & & & & & & & & \\
P.G. & -0.70 & 0.68 & 1 & & & & & & \\
& $0.03 *$ & $0.04^{*}$ & & & & & & & \\
P.M. & -0.67 & 0.87 & 0.73 & 1 & & & & & \\
& $0.04^{*}$ & $0.01 *$ & $0.03^{*}$ & & & & & & \\
C.R.T. & -0.69 & 0.92 & 0.80 & 0.85 & 1 & & & & \\
& $0.04 *$ & $0.01 *$ & 0.01 & $0.01 *$ & & & & & \\
C.G. & -0.70 & 0.68 & 1 & 0.73 & 0.81 & 1 & & & \\
& $0.03 *$ & $0.03 *$ & $0 *$ & $0.03 *$ & $0.02 *$ & & & & \\
C.M. & -0.67 & 0.84 & 0.80 & 0.91 & 0.84 & 0.80 & 1 & & \\
& $0.04 *$ & $0.01 *$ & $0.01 *$ & $0.01 *$ & $0.01 *$ & $0.02 *$ & & & \\
L.R.T. & 0.36 & -0.69 & -0.24 & -0.66 & -0.68 & -0.24 & -0.61 & 1 & \\
& 0.26 & $0.03 *$ & 0.45 & $0.04 *$ & $0.04 *$ & 0.45 & 0.06 & & \\
L.G. & 0.29 & 0.03 & 0.15 & 0.27 & 0.12 & 0.15 & 0.28 & 0.04 & 1 \\
& 0.37 & 0.92 & 0.64 & 0.41 & 0.70 & 0.64 & 0.39 & 0.89 & \\
L.M. & 0.41 & -0.27 & -0.30 & -0.01 & -0.27 & -0.30 & -0.15 & 0.26 & 0.61 \\
& 0.20 & 0.41 & 0.35 & 0.98 & 0.41 & 0.35 & 0.64 & 0.43 & 0.06 \\
\hline
\end{tabular}

P.R.T.= Proteínas resto de tejido; P.G.= Proteínas gónada; P.M.= Proteínas músculo; C.RT.= Carbohidratos resto de tejido; C.G.= Carbohidratos gónada; C.M.= Carbohidratos músculo; L.RT.= Lípidos resto de tejido; L.G.= Lípidos gónada; L.M.= Lípidos músculo; $*=(P<0.05)$ P.R.T. = remaining tissue proteins; P.G. $=$ gonad proteins; P.M. = muscle proteins; C.RT.= remaining tissue carbohydrates; C.G. = gonad carbohydrates; C.M. = muscle carbohydrates; L.RT. = remaining tissue lipids; L.G. = gonad lipids; L.M. = muscle lipids; $*=(P<0.05)$

Aubyn (1998), Rangel \& D'Lacoste (2004), Aponte et al. (2008) y Lista et al. (2008), quienes también reportaron tallas promedio mayores a $55 \mathrm{~mm}$ para la misma especie. La masa seca de los diferentes tejidos mostró los mayores valores en tallas comprendidas entre 70-78 $\mathrm{mm}$, principalmente en septiembre, diciembre, febrero y mayo, donde los organismos presentaron la mayor actividad reproductiva, lo cual coincide con lo señalado por Lista et al. (2006), quienes afirman que entre estas longitudes los organismos muestran cambios bruscos en su masa gonádica.

Los niveles de proteínas en el resto de tejido, las gónadas y el músculo presentaron una relación inversa, con respecto a la biomasa seca de la gónada, lo que podría sugerir que $A$. zebra 
utilizó las proteínas como sustrato energético durante su actividad reproductiva, ya que los incrementos de la masa seca de las gónadas venían acompañados simultáneamente por descensos de las proteínas (septiembre y diciembre de 2010, mayo de 2011). En este sentido, se ha demostrado que las proteínas, aparte de constituir uno de los principales componentes de los ovocitos en bivalvos (Gireesh et al. 2009), pueden aportar energía durante el proceso gametogénico, después de los carbohidratos y los lípidos de reserva (Barber \& Blake, 1981), lo que podría significar que las proteínas fueron utilizadas como una fuente importante de energía durante la gametogénesis en A. zebra, tal y como ha sido reportado para otras especies, tales como: Crassostrea gigas (Li et al. 2000), Crassostrea plicatula (Li et al. 2006) y Sinonovacula constricta (Yan et al. 2010).

La contribución de las proteínas procedentes del músculo durante la gametogénesis también se ha reportado para Mytilus edulis (Gabbott \& Bayne, 1973). Sin embargo, algunos estudios muestran que son las proteínas del manto las que aportan la energía necesaria para la gametogénesis (Gabbott \& Bayne, 1973; Zandee et al. 1980). En tal sentido, esto podría explicar la disminución del contenido proteico en el resto de tejido cuando la biomasa seca gonadal aumenta, ya que en dicho tejido se encuentra inmerso el manto. En las gónadas también se observó un descenso notable de las proteínas relacionado con posibles desoves. La expulsión de los gametos y tejido gonádico podría explicar dichas caídas como ha sido señalado por Acosta et al. (2010) en mejillones.

Los carbohidratos, al igual que las proteínas, mostraron las menores concentraciones en el periodo comprendido entre septiembre-diciembre de 2010 y las máximas en mayo y julio de 2011, coincidiendo con temperaturas bajas y aumentos en la clorofila $a$ en la zona, siendo estos factores ambientales los que explicaron el $61.5 \%$ y $57.4 \%$ de la variación observada, solamente para el resto de tejido y el músculo. Sin embargo, la relación porcentual entre carbohidratos y proteínas suele ser inversa tal y como ha sido reportada en: Polymesoda radiata (Ruiz et al. 1998), Mytilus galloprovincialis por Bressan \& Marín (1985), Mytilus edulis (Dare \& Edwards, 1975), Crassostrea iridescens y C. corteziensis por Páez-Osuna et al. (1993) y Tagelus peruvianus (Fonseca et al. 2011).

En el músculo aductor se registraron los mayores valores de carbohidratos, lo que concuerda con los resultados obtenidos para el ostión Hyotissa hyotis y los mejillones Perna perna y Perna viridis (Rodríguez-Astudillo et al. 2007; Acosta et al. 2010) y para A. zebra (Sarkis, 1993). Ansell (1972) señaló que en Donax vittatus el músculo aductor es el principal órgano de almacenamiento de reservas energéticas, las cuales pueden ser movilizadas principalmente hacia las gónadas. Del mismo modo, la variación observada de los carbohidratos en el resto de tejidos puede ser atribuible al hecho de que dentro de dichos tejidos se encontraba unida la glándula digestiva, la cual ha sido señalada por Natera (2010), Her- 
nández (2012) y Ren et al. (2003) como un posible órgano de reservas energéticas y que también podrían ser utilizadas durante la actividad reproductiva.

La posible movilización de los carbohidratos totales entre los diferentes tejidos (resto, músculo y gónada) durante el proceso gametogénico se evidenció al mostrarse una correlación inversa con la biomasa seca gonadal para soportar la gametogénesis; de hecho, diversos estudios indican que los carbohidratos constituyen la principal fuente de energía en bivalvos como: Crassostrea iridescens y C. corteziensis (PáezOsuna et al. 1993), Crassostrea gigas (Ren et al. 2003), Mytilus edulis $\mathrm{y}$ Mytilus galloprovincialis (Kopp et al. 2005). Esto explica el posible uso en conjunto de las proteínas y los carbohidratos como fuente de energía.

Con base en lo anterior, se sugiere que durante los procesos reproductivos, A. zebra utiliza como mecanismo energético la tercera vía señalada por Acosta et al. (2010), correspondiente a la utilización y almacenamiento en órganos especializados de la energía una vez obtenida del alimento, por lo que $A$. zebra emplearía la transferencia y conversión de energía sobre todo de las reservas contenidas en el músculo y el resto de tejido, y como alternativa, la dependencia relativamente directa del suministro y disponibilidad de alimento en el medio. Manteniendo, de esta manera, un posible equilibrio entre las proporciones de los tres componentes bioquímicos, evitando un desgaste energético extremadamente alto que podría comprometer su estabilidad fisiológica.

Los lípidos no manifestaron diferencias con respecto a su variación mensual. Solamente, los niveles de lípidos cuantificados en el resto de tejido presentaron una asociación con la clorofila $a$ con un $53.8 \%$ de la varianza observada; sin embargo, la clorofila $a$ mostró un coeficiente negativo, lo que evidenció la relación inversa entre este parámetro y la energía contenida en los lípidos, lo que podría sugerir que el anabolismo y catabolismo de los lípidos depende de rutas metabólicas conservativas, menos influenciables por variaciones ambientales, pero sensibles al contenido interno de otros sustratos energéticos, como ha sido indicado por Rodríguez-Astudillo et al. (2007).

La falta de explicación de la varianza observada en los contenidos de lípidos de las gónadas podría ser atribuida a varios factores, entre los que podemosnombrar: 1)Alprobable efecto amortiguador de la neolipogénesis a partir de los carbohidratos de otros tejidos de reservas estudiados, lo que probablemente mantuvo los valores en un rango relativamente estable (correlación inversa entre carbohidratos y masa gonádica). 2) Al efecto enmascarador de una asincronía reproductiva de la población, tal y como fue reportado por Lista et al. (2011). 3) A la perturbación en los análisis causada por los procesos de desove que afectarían de forma independiente una caída en los contenidos de lípidos de las gónadas, de manera independiente de la disponibilidad de alimento 
(estrategia conservativa u oportunista) o en diferentes niveles de temperatura. Para demostrar la afirmación anterior, se hace necesario incluir análisis que relacionen los contenidos lipídicos con distintas etapas reproductivas, dado que se ha reportado que los estadios reproductivos de algunos bivalvos, como mejillones, pueden explicar más del $40 \%$ de la varianza observada en los contenidos de carbohidratos y lípidos de gónadas y músculo aductor (Acosta et al. 2010).

Arca zebra presentó un aumento de sus componentes bioquímicos basado sobre todo en la variación y/o disponibilidad de alimento (estimado como clorofila a); sin embargo, los carbohidratos y las proteínas fluctuaron posiblemente en repuesta a las necesidades energéticas que presentó este bivalvo a los procesos reproductivos. Los datos obtenidos en este estudio (talla, peso seco total $\mathrm{y}$ componentes bioquímicos) sugieren que $A$. zebra muestra un excelente estado nutricional para su comercialización e ingesta, particularmente durante los periodos septiembre-diciembre y marzo-junio. No obstante, hay que tomar en cuenta los posibles periodos de máxima producción de gametos encontrados en septiembre, diciembre y mayo, por lo que se recomienda como estrategia establecer como época preventiva de extracción los periodos que van de febrero-marzo, junio-julio y octubrenoviembre, que no comprometen las épocas de mayor actividad reproductiva (desove), asegurando así una mayor tasa de reclutamiento y mantenimiento de los bancos naturales a lo largo del tiempo, lo cual permitiría un mejor aprovechamiento sustentable y una explotación racional de este rubro pesquero.

\section{AGRADECIMIENTOS}

Los autores desean expresar su agradecimiento al Consejo de Investigación de la Universidad de Oriente, por el financiamiento del proyecto: Variación estacional de la composición bioquímica de la pepitona Arca zebra, en relación con el estadio reproductivo y los factores ambientales ( ${ }^{\circ}$ CI-0203060 I-177712); así como a los evaluadores por sus valiosas observaciones.

\section{BIBLIOGRAFÍA}

Acosta, V., Prieto, A., Ruiz, L. \& Gil, H. (2007). Moluscos asociados a la pepitona Arca zebra (Mollusca: Bivalvia) en Chacopata, estado Sucre, Venezuela. Saber, 19(1), 21-26.

Acosta, V., Natera, Y., Lodeiros, C., Freites, L. \& Vásquez, A. (2010). Componentes bioquímicos de los tejidos de Perna perna y $P$. viridis (Lineo, 1758) (Bivalvia: Mytilidae), en relación al crecimiento en condiciones de cultivo suspendido. Lat. Am. J. Aquat.Res., 38(1), 37-46. doi: 10.3856/ vol38-issue1-fulltext-4.

Acuña, A. (1977). Fijación, crecimiento y composición química de la pepitona Arca zebra en la región oriental de Venezuela. Trabajo para ascender a la categoría de Profesor Agregado no publicado. Instituto Oceanográfico, Universidad de Oriente, Cumaná. 
Ansell, A. D. (1972). Distribution, growth and seasonal changes in biochemical composition for the bivalve Donax vittatus ( $\mathrm{Da}$ Costa) from Kames Bay, Milport. J. Exp. Mar. Biol. Ecol., 10, 137-150. doi: 10.1016/0022-0981(72)90099-8.

Aponte, A., Prieto, A. \& Lemus, M. (2008). Relación longitud-peso seco de la pepitona Arca zebra (Swainson, 1833) procedente de la costa norte de la Península de Araya, Estado Sucre, Venezuela. Bol. Inst. Oceanogr. Ven., 47(1), 59-65.

Arias, A. (1976). Algunos aspectos bioquímicos y tecnológicos en la especie Arca zebra (Swainson, 1833). Tesis de pregrado no publicada. Departamento de Biología, Universidad de Oriente, Cumaná.

Barber, B. \& Blake, N. (1981). Energy storage and utilization in relation to gametogenesis in Argopecten irradians concentricus (Say). J. Exper. Mar. Biol. Ecol., 52, 121-134. doi: 10.1016/0022-0981(81)90031-9.

Bligh, E. \& Dyer, W. (1959). A rapid method of total lipid extraction and purification. Can J. Biochem Physiol., 37, 911-917. doi: 10.1139/059-099.

Bradford, M. (1976). A rapid and sensitive method for the quantitation of microgram quantities of protein utilizing the principle of protein-dye binding. Anal. Chem., 72, 248-254. doi: 10.1016/0003-2697(76)90527-3.

Bressan, M. \& Marín, M. (1985). Seasonal variations in biochemical composition and condition index of cultured mussels Mytilus galloprovincialis (LMK) in the lagoon of Venice (North Adriatic). Aquacult., 48, 13-21. doi: 10.1016/0044-8486(85)90048-1.

Cabello, A., Villarroel, R., Figuera, B., Ramos, M., Márquez, Y. \&
Vallenilla, M. (2004). Parámetros de frescura de moluscos. Rev. Cient. FCV-LUZ, 5, 457-466.

Dare, P. \& Edwards, D. (1975). Seasonal changes in flesh weight and biochemical composition of mussels (Mytilus edulis L.) in the Conwy estuary, north wales. J. Exp. Mar. Biol. Ecol., 18, 89-97. doi: 10.1016/0022-0981(75)90066-0.

Dubois, M., Guilles, K., Hamilton, K., Rebers, P. \& Smith, F. (1956). Colorimetric method for determination of sugars and related substances. Anal. Chem., 28, 350356. doi: 10.1021/ac60111a017.

Fonseca, C., Marín, C., Chavarría, F., Cruz, R. \& Toledo, P. (2011). Variación estacional de la composición proximal del mejillón Tagelus peruvianus (Bivalvia: Solecurtidae) del Golfo de Nicoya, Puntarenas, Costa Rica. Rev. Biol. Trop., 59(4), 1517-1523.

Gabbott, P. (1983). Developmental and seasonal metabolic activities in marine mollusks. In P. Hochachka (Ed.), The Mollusca 2: environmental biochemistry and physiology (pp. 165-217). New York, EE. UU.: Academic Press.

Gabbott, P. \& Bayne, B. (1973). Biochemical effects of temperature and nutritive stress on Mytilus edulis L. J. Mar. Biol. Assoc. U.K., 53, 269-286. doi: 10.1017/S0025315400022268.

Gireesh, R., Biju, A. \& Muthiah, P. (2009). Biochemical changes during larval development in the short neck clam, Paphiama labarica Chemnitz. Aquacult. Res., 40, 1510-1516. doi: 10.1111/j.1365-2109.2009.02251.x.

Hair, J., Anderson, R., Tatham, R. \& Black, W. (1992). Multivariate data analysis. New York, EE. UU.: Mac Millan Pub. CO.

Hernández, C. (2012). Efecto de la dieta en el desarrollo y composición de 
la gónada y tejidos somáticos de la concha nácar Pteria sterna (Gould, 1851). Tesis de postgrado en Ciencias en el uso, manejo y preservación de los recursos naturales no publicada. Centro de Investigaciones Biológicas del Noroeste, S.C., México.

Kopp, J., Cornette, F. \& Simonne, C. (2005). A comparison of growth and biochemical composition Mytilus galloprovincialis (Lmk.) and Mytilus edulis (L.) on the West coast of Cotentin, Normandy, France 19992000. Aquac. Inter, 13(4), 327-340.

Li, Q., Liu, W., Shirasu, K., Chen, W. \& Jiang, S. (2006). Reproductive cycle and biochemical composition of the Zhe oyster Crassostrea plicatula (Gmelin) in an eastern coastal bay of China. Aquacult., 261, 752-759. doi: 10.1016/j.aquaculture.2006.08.023.

Li, Q., Osada, M. \& Mori, K. (2000). Seasonal biochemical variations in Pacific oyster gonadal tissue during sexual maturation. Fish. Scien., 66, 502-508. doi: 10.1046/j.1444-2906.2000.00067.x.

Lista, M., Lodeiros, C., Prieto, A., Himmelman, J., Castañeda, J., García, N. \& Velásquez, C. (2006). Relation of seasonal changes in the mass of the gonad and somatic tissues of the zebra ark shell Arca zebra to environmental factors. J. Shellfish. Res., 25, 969-973. doi: 10.2983/0730-8000(2006)25[969:ROS CIT]2.0.CO;2.

Lista, M., Prieto, A., Velásquez, C., Lodeiros, C. \& Hernández, G. (2008). Variación mensual del índice de condición y madurez sexual en la pepitona Arca zebra, del banco de Chacopata, Península de Araya, estado Sucre, Venezuela. Saber, 20(1), 29-38.
Lista, M., Prieto, A., Velásquez, C., Lodeiros, C., Acosta, V., Longart, Y. \& Hernández, G. (2011). Descripción y variación mensual de las etapas reproductivas de la pepitona Arca zebra (Swainson, 1833) en el banco de Chacopata, península de Araya, estado Sucre, Venezuela. Zootec. Trop., 29(1), 89-102.

Mendoza, J. (1999). Análisis de la pesca artesanal maritima en Venezuela: situación actual y perspectivas. Caracas, Venezuela: Instituto Interamericano de Cooperación para la Agricultura. Organización de Estados Americanos.

Mendoza, J. (2008). Situación actual $\mathrm{y}$ perspectivas de las pesquerías marítimas en Venezuela. Rec. Mar. Acui., 2, 121-137.

Natera, Y. (2010). Composición bioquímica de los mejillones Perna perna y $P$. viridis bajo sistema de cultivo suspendido en la ensenada de Turpialito. Trabajo de pregrado para obtener el título de licenciatura no publicado. Departamento de Biología, Universidad de Oriente, Cumaná.

Páez-Osuna, F., Zozueta, H. \& Osuna, J. (1993). Biochemical composition of the oysters Crassostrea iridescens (Hanley) and Crassostrea corteziensis (Hertlein) in the north-west coast of Mexico: Seasonal changes. $J$. Exp. Mar. Biol. Ecol., 170, 1-9. doi: 10.1016/0022-0981(93)90125-8.

Pande, S., Khan, R. \& Venkitsubra, T. (1963). Microdetermination of lipids and serum total fatty acids. Anal. Biochem., 6, 415-423. doi: 10.1016/0003-2697(63)90094-0.

Pérez, A., Delgado, M., Fernández, M. \& Labarta, U. (2003). Energy balance, gonad development and biochemical composition in the clam Ruditapes 
decussatus. Mar. Ecol. Prog. Ser., 258, 133-145. doi: 10.3354/meps258133.

Prieto, A. \& Saint-Aubyn, M. (1998). Crecimiento del bivalvo Arca zebra (Swainson, 1883), en Chacopata, estado Sucre, Venezuela. Saber, 10, 14-19.

Rangel, J. \& D'Lacoste, T. (2004). Pesquería y aprovechamiento de la pepitona en la playa El Turco, estado Sucre. INIA, 1, 23-26.

Ren, J., Marsden, I., Ross, A. \& Schiel, D. (2003). Seasonal variation in the reproductive activity and biochemical composition of the Pacific oyster (Crassostrea gigas) from the Marlborough Sounds, New Zealand. New Zealand J. Mar. Fresh. Res., 37, 171-182. doi: 10.1080/00288330.2003.9517155.

Rodríguez-Astudillo, S., Villalejo-Fuerte, M., García-Domínguez, F., GuerreroCaballero, R. \& Ramírez-Sevilla, R. (2007). Composición bioquímica del ostión Hyotissa hyotis (Linnaeus, 1758) durante La Niña 1998-1999 y El Niño 1997-1998 en el Golfo de California. Rev. Biol. Mar. Oceanogr., 42(3), 393-402. doi: 10.4067/ S0718-19572007000300019.
Ruiz, E., Cabrera, J., Cruz, R. \& Palacios, J. (1998). Composición bioquímica de la carne de Polymesoda radiata (Bivalvia: Corbiculidae) en Costa Rica. Rev. Biol. Trop., 46(3), 649-653. Sarkis, S. (1993). Seasonal changes in the gross biochemical composition of the turkey wing Arca zebra, in Bermuda. J. Shellfish. Res., 12(2), 329-336.

Statpoint. (2006). Statgraphics Centurion $X V$. Statistical exploration and modeling software. Virginia, EE. UU.: Statpoint Technologies, Inc.

Strickland, J. \& Parsons, T. (1972). A practical handbook of seawater analysis. Bull. Fish. Rev., 16, 167-315. Yan, H., Li, Q., Liu, W., Yu, R. \& Kong, L. (2010). Seasonal changes in reproductive activity and biochemical composition of the razor clam Sinonovacula constricta (Lamarck 1818). Mar. Biol. Res., 6, 78-88. doi: 10.1080/17451000903039756.

Zandee, D., Kluytmans, J., Zurburg, W. \& Pieters, H. (1980). Seasonal variations in biochemical composition of Mytilus edulis with reference to energy metabolism and gametogenesis. Neth. J. S. Res., 14, 1-29. doi: 10.1016/0077-7579(80)90011-3.

Zar, J. (2010). Biostatistical Analysis. (2da. ed.). New Jersey, EE. UU.: Prentice-Hall. 